\title{
THE SIMPLEST NOTCH AND BANDSTOP FILTERS BASED ON THE SLOTTED STRIPS
}

\author{
A. Kirilenko *, L. Mospan ${ }^{\infty}$ \\ * Institute of Radiophysics and Electronics of the National Academy of Sciences of Ukraine \\ 12, Ac. Proskury Str., 61085, Kharkov, Ukraine \\ phone: 380572 448518, fax: 380572 441105, 380572 124829, E-mail kirilenko@ire.kharkov.ua \\ ${ }^{\infty}$ Institute of Radiophysics and Electronics of the National Academy of Sciences of Ukraine \\ 12, Ac. Proskury Str., 61085, Kharkov, Ukraine \\ phone: 380572 448428, fax: 380572 441105, E-mail lyuda@ire.kharkov.ua
}

\begin{abstract}
New configuration of the notch and bandstop filters is proposed and investigated. Here a rejection section is based on a thin slotted H-plane strip that may be considered as a limiting case of a threeslot iris. Such a section forms symmetrical frequency response with very low insertion loss out of the stopband. The results of numerical design and measurement data are given for a 3\% notch filter and for $2 \%$ three-section bandstop one. In the low-power applications the proposed notch filter is probably the simplest one due to its easy fabrication and a possibility to be incorporated into a waveguide circuit by placing between the waveguide flanges.
\end{abstract}

The property of a multi-slot iris to form a frequency response with a point of total reflection was initially discussed by Paterson and Anderson (1) on the basis of circuit theory considerations. Later on the treatment using the eigen-oscillations of open waveguide circuit was used by Kirilenko and Mospan (2) to explain the nature and the main features of such rejection phenomena. In particular it was shown that the reason of such a rejection resonance is simultaneous excitation of the low-Q and high-Q eigen-oscillations and even two different slots can provide the existence of rejection point. As it turned out, rectangular slot shape isn't obligatory. As example, a two-slot iris with elliptical slot apertures, discussed by C.Tomassoni et. al. (3), provides the existence of rejection point as well. Some of configurations of band-rejection filters based on the multi-slot irises were demonstrated by Kirilenko et. al. (4).

What all the rejection irises (1-4) have in common is a relatively large insertion loss in the whole passband or in its essential part. It turned out that there is a limiting configuration of a three-slot iris that provides very small insertion loss out of the stopband. That is achieved practically in the whole operating range of a rectangular waveguide and up to the second resonance point located in the twoor three-mode frequency band. The geometry of such a three-slot iris is very simplest (Fig.1): it has two slots, upper and lower, extended to waveguide walls and. in fact, it is an $H$-plane strip with a longitudinal slot. Therefore we shall call it further the "slotted strip". In the numerical modeling of such a waveguide circuit it is considered as a combination of two plane junctions separated by a small section of three parallel waveguides. Mode-matching based key element and S-matrix technique provide an efficient solution.

The calculated frequency response of a slotted strip symmetrically placed in the cross-section of the waveguide WR90 is shown in Fig. 1 by the solid curve. The slot width $a 1$ and height $b 1$ are respectively 13.7 and 1.0 (all the dimensions are in $\mathrm{mm}$ ), the height $d$ of metal bridges bounding 
the slot is 1.0 and the strip (three-slot iris) thickness $t$ is 0.5 . Such a waveguide element has a classical response of a lumped rejection circuit, that is symmetrical with respect to the rejection frequency and has very low insertion loss $L_{p}$ out of the stopband: $L_{p}<0.05 d B$ for the frequencies lower than $9.67 \mathrm{GHz}$ and upper than $11.4 \mathrm{GHz}$. The calculated frequency of rejection resonance is $f_{r e j}=10.69 \mathrm{GHz}$. The bandwidth of the resonance, defined by the $3 \mathrm{~dB}$ level, is $3 \%$. The measured insertion loss at the central frequency reaches $35 \mathrm{~dB}$. Some disagreement between the calculated and the measured data in the upper part of the frequency range is connected to the measurement equipment limitations, not to the measured inhomogeneity.

The comulative information about the slotted strip "possibilities" to provide the specified Q-factor and placement of resonance is presented in Fig.2 in the form of equal-level curves $Q=$ const and $f_{r e j}=$ const in the coordinates "bridge height and slot width" for a fixed slot height. First of all note that the location of the resonance is determined mainly by the width of the slot, that is close to halflambda in the resonance. Hence, the rejection may be provided at any frequency point, including a part of two-mode frequency range. The quality factor of rejection resonance depends primarily on the bridge and the slot heights. Decreasing the bridge heights $d$ with a constant slot height $b 1$ results in increasing the resonance Q-factor. A similar dependence is observed on the slot height with a constant bridge height. That is the same as for transmission resonances in conventional resonance irises: the narrower iris slot, the higher quality of the resonance. However here we deal with the resonance of total reflection. The bridge and slot heights are restricted by certain fabrication and electric breakdown limitations. If we choose, for example, fixed $d$ equal to the fabrication limit of $0.15 \mathrm{~mm}$, then the resonance with $Q \leq 50$ can be provided easily at any frequency in the whole operating range by choosing an appropriate slot height from 4 to 5 (in the lower part of operating band) and up to 1.0 (near its end). On the other hand with a given restriction on $d$ it is impossible to reach $Q \approx 100$ at high frequencies with $b 1 \geq 1.0$, and we are forced to choose narrower slots. For example, the quality more 100 can be reached within the upper part of operating range with the above-mentioned minimum $d$ and $b 1<0.2 \div 0.3$ only. Here we have a chance to violate power (or fabrication) restrictions in terms of the distance between the bridges. Fig. 3 contains some additional information about the relation between the slot dimensions and bridge height providing required $Q$-factor. It contains the plots, which describe the coupling between $d$ and $a 1$ the for given $Q=25$. The resonant frequency varies along each curve with a fixed slot height $b 1$.

The amplitudes of the fields that are excited in the slotted strip at the total reflection point are interesting. Field amplitudes in the strip slot and the top and the bottom openings are very different. Field amplitudes in the top and the bottom openings are several times as large as one of the incident wave, whereas the field amplitude in the strip slot are more by a factor of $10 \times 2$. Some examples for the slotted strip of the thickness of $0.5 \mathrm{~mm}$ in WR90 are given in the Table 1.

As an application of slotted strips, the three-section Chebyshev bandstop filter with the $10.25 \mathrm{GHz}$ center frequency, $2 \%$ stopband and $0.5 d B$ passband ripples was designed and tested. Its dimensions are given in Table 2. The calculated and measured responses of such a filter are shown in Fig. 4. Some disagreement in stopband placement and out-of-stopband insertion loss may be related to fabrication errors. Used measuring equipment enabled us to find that the stopband insertion loss exceed $35 \mathrm{~dB}$. Both fronts of the stopband have high skirt selectivity. 
The wideband response given in Fig. 5 shows an excellent out-of-band performance. For a comparison, return loss of a bandstop filter based on the E-plane stubs (5) is given in the same figure. The second spurious stopband appears near $30 \mathrm{GHz}$ for the slotted strip filter, whereas it is near $25 \mathrm{GHz}$ for the filter based on the E-plane stubs. Approximately 30\% broadening of the upper passband is achieved with the slotted strip filter.

\section{CONCLUSION}

New configuration of the waveguide bandstop filters based on the metal slotted strips placed in a rectangular waveguide has very good performance in the low-power applications. Such filters incorporate following attracting features: they have short longitudinal dimensions and do not exceed transversal dimensions of a waveguide; the filters are low-cost and easy-to-fabricate; corresponding notch filter can be easily introduced into any waveguide circuit by placing a slotted strip iris between he waveguide flanges.

\section{REFERENCES}

1. N.G.Paterson, I.Anderson, Bandstop iris for rectangular waveguide, Electronics Letters, 1976, Vol.12, №22, pp.592-594.

2. A.A.Kirilenko, L.P.Mospan, Reflection resonances and natural frequencies of two-aperture iris in rectangular waveguide, IEEE Trans. on MTT, 2000, Vol.48, No.8, pp.1419-1421.

3. C.Tomassoni, M.Mongiardo, L.Tarricone, Analytical Evaluation of coupling integrals for elliptical irises in rectangular waveguide, Proc of 30th Eur. Microwave Conf., Paris, France, 3-5 Oct., 2000, Vol.2, pp.222-225.

4. A.A.Kirilenko, L.P.Mospan, Two- and three- slot irises as the bandstop filter sections, Microwave and Opt. Tech. Letters, 2001, Vol.28, No.4, pp.282-284.

5. A.A Kirilenko, L.A.Rud', S.L.Senkevich, Spectral approach to the synthesis of bandstop filters, IEEE Trans. on MTT, 1994, Vol.42, N7, pp.1387-1392.

Table 1. Slotted strip field amplitudes

\begin{tabular}{|c|c|c|c|c|}
\hline \multicolumn{2}{|c|}{ Slotted strip dimensions } & \multirow{2}{*}{$Q$-factor } & \multicolumn{2}{|c|}{ Field amplitudes * } \\
\cline { 4 - 5 } & & & in the slot & in the openings \\
\hline $13.5 \times 2.0$ & 2.0 & 10 & 1.7 & 350 \\
\hline $13.5 \times 2.0$ & 1.0 & 20 & 3 & 470 \\
\hline $13.5 \times 1.0$ & 1.0 & 30 & 4 & 380 \\
\hline
\end{tabular}

(*They are normalized to the incident wave amplitude in the center of the input waveguide).

Table 2. Bandstop filter geometry

\begin{tabular}{|c|c|c|c|c|c|}
\hline \multicolumn{2}{|c|}{ 1st and 3rd slotted strips } & \multicolumn{2}{|c|}{ 2nd slotted strip } & Strip thickness, & WR90 sections, \\
\cline { 1 - 3 }$a 1 \times b 1, \mathrm{~mm}^{2}$ & $d, \mathrm{~mm}$ & $a 1 \times b 1, \mathrm{~mm}^{2}$ & $d, \mathrm{~mm}$ & $t \mathrm{~mm}$ & $l, \mathrm{~mm}$ \\
\hline $13.7 \times 2.0$ & 0.15 & $13.79 \times 1.5$ & 0.15 & 0.5 & 9.95 \\
\hline
\end{tabular}




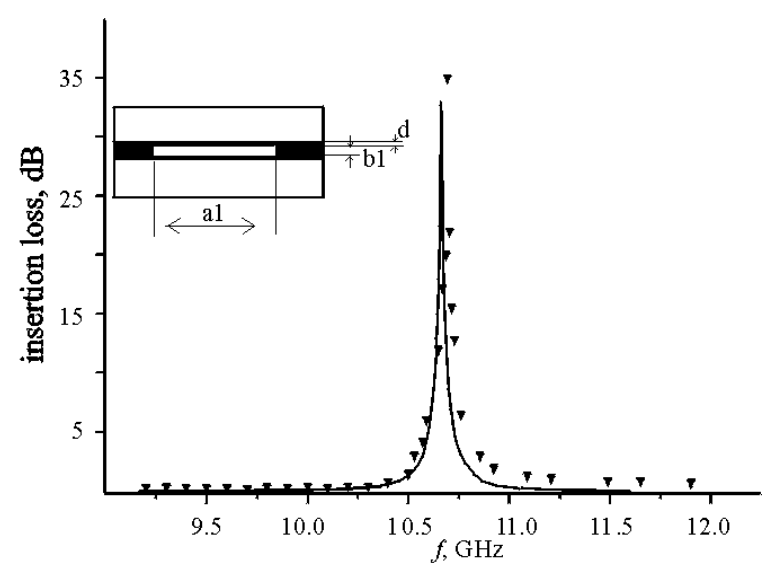

Fig. 1 Cross-section of a notch filter based on a slotted strip and insertion loss for the slotted strip with $b 1=1.0 \mathrm{~mm}, t=0.5 \mathrm{~mm}, a 1=13.7 \mathrm{~mm}$ in $W R-90$ (solid curve - calculations, triangles experiment)
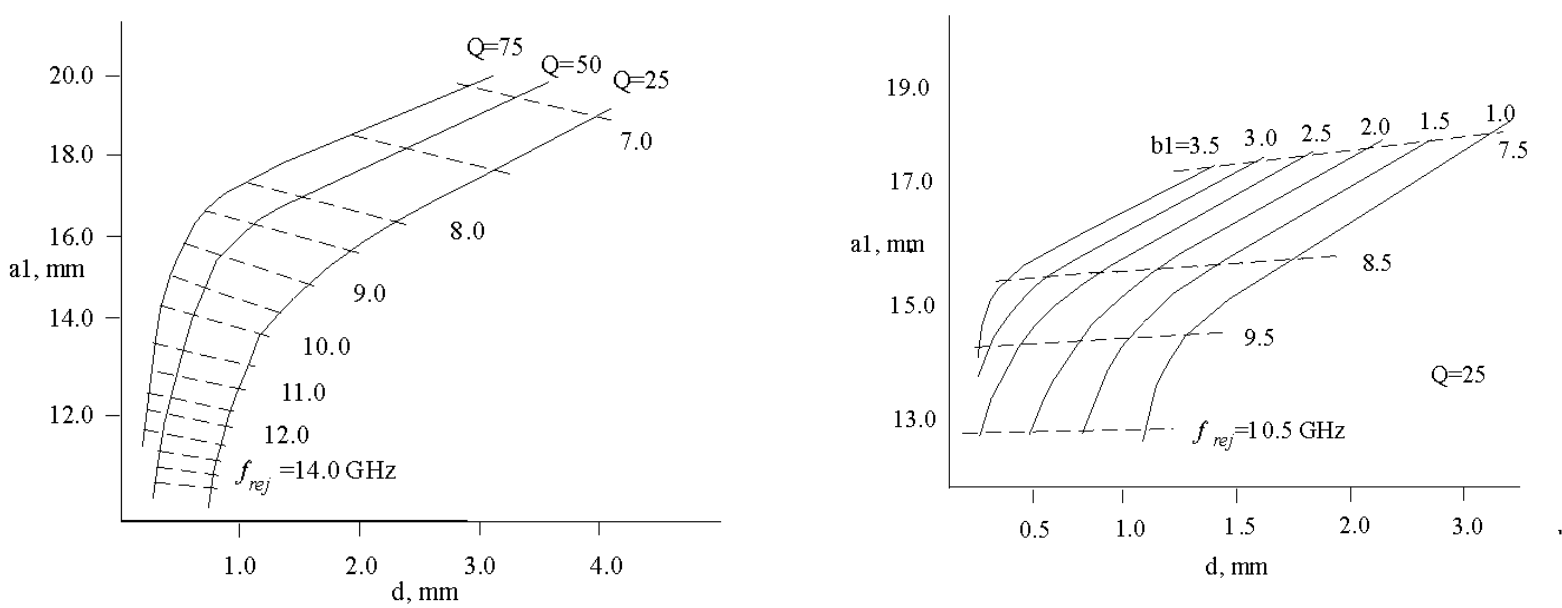

Fig.2. The curves of $Q=$ const and Fig.3 The plots, characterizing the relation $f_{\text {rej }}=$ const for a slotted strip in the waveguide $W R 90(b 1=1.0 \mathrm{~mm}, t=0.5 \mathrm{~mm})$ between the bridge heights and slot width for specified $Q=25 . \quad($ WR90,$t=0.5 \mathrm{~mm}$, different slot heights $b 1$ are marked on the curves)
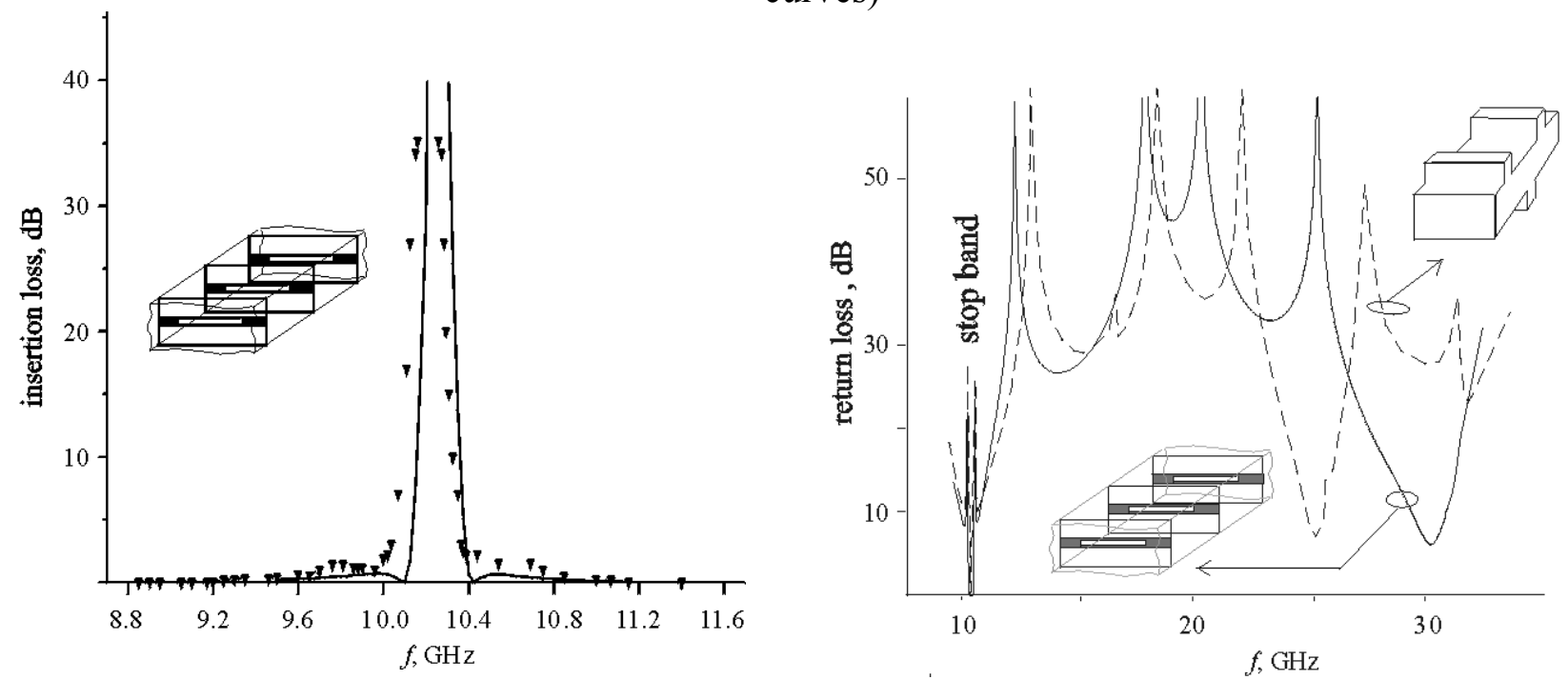

Fig 4. Insertion loss for a three section BSF based on the slotted strips

Fig 5. Return loss for a three section BSF based on the slotted strips 